\title{
Erratum to: Algebraic flux correction for nonconforming finite element discretizations of scalar transport problems
}

\section{Matthias Möller}

Published online: 16 March 2014

(C) Springer-Verlag Wien 2014

\section{Erratum to: Computing (2013) 95:425-448}

DOI 10.1007/s00607-012-0276-y

In the original publication, the Fig. 9b (left diagram) has been published incorrectly. The corrected Fig. 9 is given on the following page:

The online version of the original article can be found under doi:10.1007/s00607-012-0276-y.

M. Möller $(\varangle)$

Institute of Applied Mathematics (LS III), TU Dortmund University of Technology,

Vogelpothsweg 87, 44227 Dortmund, Germany

e-mail: matthias.moeller@math.tu-dortmund.de 
(a) Dual-socket Intel Xeon quad-core X5550
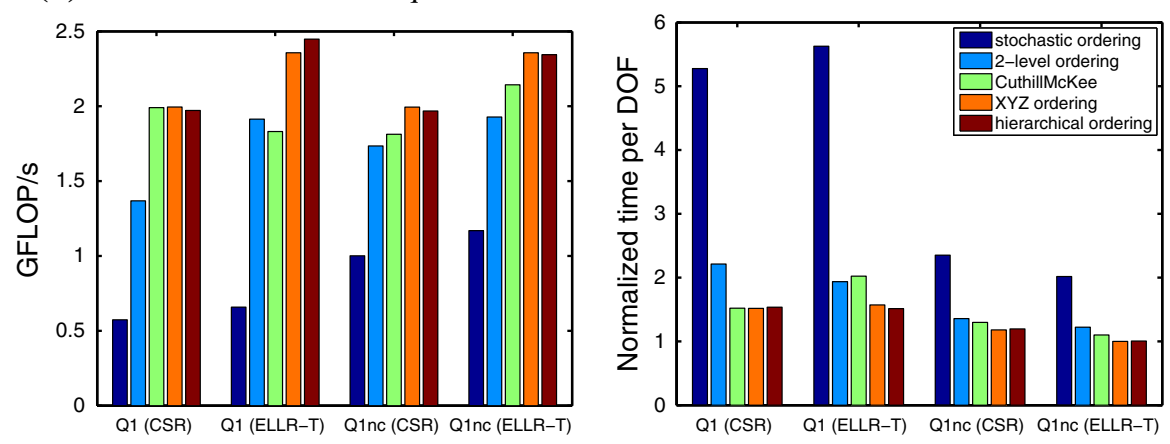

(b) Tesla C2070 GPU (ECC off)
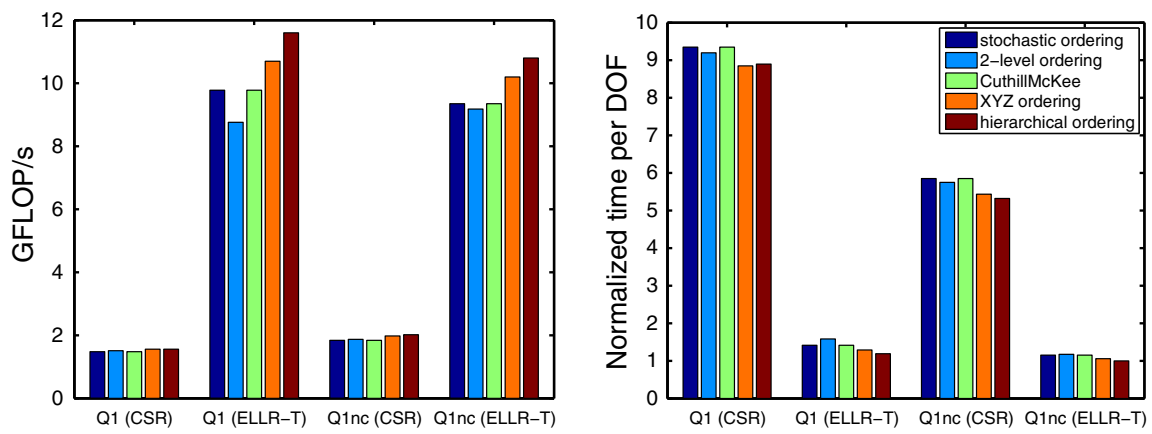

Fig. 9 Comparing the performance of SpMV for finite element matrices stored in CSR and ELL format on CPUs and CSR and ELLR-T format on GPUs 